Results DEMOGRAPHY: 528 subjects were colonoscoped at one single centre. The aspirin group $(n=112)$ had $74(66 \%)$ males and a median age of 68 years. The NSAID group $(n=46)$ had $23(50 \%)$ males and a median age of 58 years. The control group, not using either of these agents $(n=370)$, had $207(56 \%)$ males and a median age of 63 years ( $p<0.001$ vs aspirin users; $p=0.012$ vs NSAID users). POLYPS: ASPIRIN vs CONTROLS. Polyps $>5 \mathrm{~mm}$ were found in $21(19 \%)$ subjects taking aspirin vs 99 (27\%) controls [odds ratio, OR, 0.57; 95\% confidence intervals, CI, (0.33-0.98); $\mathrm{p}=0.042]$. Polyps $>5 \mathrm{~mm}$ or cancer were found in $24(21 \%)$ aspirin users vs 118 (32\%) controls [OR, 0.50; 95\% CI (0.30.0.84); $\mathrm{p}=0.009]$. POLYPS: NSAIDs vs CONTROLS. Polyps $>5 \mathrm{~mm}$ were found in $5(11 \%)$ subjects taking NSAIDs vs $99(27 \%)$ controls [OR, 0.36; 95\% CI (0.14-0.86); $\mathrm{p}=0.036]$. Polyps $>5 \mathrm{~mm}$ or cancer were found in $6(13 \%)$ NSAID users vs 118 (32\%) controls [OR, 0.35; 95\% CI $(0.14-0.86) ; p=0.022$.

Conclusions (1) In this bowel cancer screening program, subjects taking low-dose aspirin were older while those taking NSAIDs were younger than controls not using either of these agents. (2) After adjusting the odds ratios for age and sex, the use of aspirin or NSAIDs was associated with lower prevalence of dysplastic colonic polyps or cancers.

These results provide further evidence for the potential use of aspirin or NSAIDs for bowel cancer chemoprevention.

\section{PTH-067 HIGH QUALITY COLON CLEANSING WITH NOVEL 1L PEG-BASED BOWEL PREPARATION NER1006 VERSUS CURRENT ALTERNATIVES}

${ }^{1}$ Richard Ng, ${ }^{1}$ Cesare Hassan, ${ }^{1}$ Jonathan Manning, 2,3,4,5,6 Michael Epstein. ${ }^{1}$ Norgine, Harefield, UK; ${ }^{2}$ Aspire Bariatrics; ${ }^{3}$ Eli Lilly; ${ }^{4}$ Zx Pharma; ${ }^{5}$ Ferring; ${ }^{6}$ Otsuka

\subsection{6/gutjnl-2018-BSGAbstracts.88}

Introduction Effective colonoscopy requires successful bowel cleansing. A segmental score of 2 on the Boston Bowel Preparation Scale (BBPS) is considered adequate for the detection of polyps $>5$ mm. ${ }^{1}$ New data indicate however that lesion detection rates may improve further at higher levels of cleansing. ${ }^{2}$

Methods The NER1006 phase 3 studies had randomised patient populations and treatment-blinded central readers for standardised BBPS assessment in the overall colon [3-5]. This post hoc analysis of all three phase 3 trials compares high versus adequate cleansing quality with NER1006 versus its comparators, using the per protocol (PP) population. Patients $(n=1521)$ aged $18-85$ years took their bowel preparations either on the day before colonoscopy (DAYB), as overnight split dosing (NOCT and MORA), or in the morning of the day of colonoscopy (MORA).

All analyses were carried out using the statistical package $R$ v3.1.3. Confidence intervals and t-test for each mean difference and two-sided P-values were calculated using Fisher's exact test.

\begin{tabular}{|c|c|c|c|c|c|c|c|}
\hline \multicolumn{8}{|c|}{ High Quality BBPS Cleansing Success (BBPS $\geq 2$ ) in the Overall Colon and Right Colon: } \\
\hline \multirow{2}{*}{$\begin{array}{l}\text { Study } \\
\text { Dosing regimen }\end{array}$} & \multicolumn{2}{|r|}{ DAYB } & \multicolumn{3}{|c|}{ MORA } & \multicolumn{2}{|c|}{ NOCT } \\
\hline & Day Before Evening & Day Before Morning/Afternoon & Overnight Split & Same Day Morning Only & Overnight Split & Overnight Split & Overnight Split \\
\hline Bowel preparation & NER1006 & $\mathrm{NaP}+\mathrm{MgCit}$ & NER1006 & NER1006 & 2L PEG+Asc & NER1006 & TS \\
\hline Patients, N & 172 & 207 & 220 & 218 & 232 & 233 & 239 \\
\hline \multicolumn{8}{|c|}{ Patients with Overall Bowel Cleansing Success, $n(\%)$} \\
\hline \multirow[t]{2}{*}{ All Segments BBPS 2-3 } & 111 & 100 & 211 & 200 & 208 & 208 & 210 \\
\hline & (64.5) & (48.3) & (95.9) & (91.7) & (89.7) & (89.3) & (87.9) \\
\hline 1 or More Segments & 20 & 6 & 82 & 83 & 57 & 76 & 84 \\
\hline BBPS 3 & (11.6) & (2.9) & (37.3) & (38.1) & (24.6) & (32.6) & (35.1) \\
\hline 2 or More Segments & 9 & 2 & 53 & 54 & 40 & 51 & 51 \\
\hline BBPS 3 & $(5.2)$ & (1) & (24.1) & (24.8) & $(17.2)$ & (21.9) & (21.3) \\
\hline \multirow[t]{2}{*}{ All 3 Segments BBPS 3} & 2 & 0 & 33 & 37 & 23 & 28 & 29 \\
\hline & $(1.2)$ & (0) & $(15.0)$ & $(17.0)$ & (9.9) & $(12.0)$ & (12.1) \\
\hline All Segments BBPS 2-3: P-Value & $<0.01$ & - & 0.01 & 0.52 & - & 0.67 & - \\
\hline 1 or More Segments & $<0.01$ & - & $<0.01$ & $<0.01$ & - & 0.63 & - \\
\hline \multicolumn{8}{|l|}{ BBPS 3: P-Value } \\
\hline 2 or More Segments & 0.03 & - & 0.08 & 0.06 & - & 0.91 & - \\
\hline \multicolumn{8}{|l|}{ BBPS 3: } \\
\hline \multicolumn{8}{|l|}{ P-Value } \\
\hline All 3 Segments BBPS 3: & 0.21 & - & 0.12 & 0.04 & - & 1.00 & - \\
\hline \multicolumn{8}{|l|}{ P-Value } \\
\hline \multicolumn{8}{|c|}{ High Quality BBPS Cleansing Success (BBPS $\geq 2$ ) in the Right Colon: } \\
\hline \multirow[t]{2}{*}{ Segmental BBPS 2-3 } & 120 & 107 & 215 & 207 & 217 & 219 & 217 \\
\hline & (69.8) & $(51.7)$ & (97.7) & $(95.0)$ & (93.5) & $(94.0)$ & $(90.8)$ \\
\hline \multirow[t]{2}{*}{ Segmental BBPS 3} & 8 & 1 & 54 & 54 & 32 & 53 & 53 \\
\hline & (4.7) & $(0.5)$ & (24.5) & (24.8) & (13.8) & (22.7) & $(22.2)$ \\
\hline BBPS 2-3: P-value & $<0.01$ & - & 0.04 & 0.55 & - & 0.23 & - \\
\hline BBPS 3: P-Value & 0.01 & - & $<0.01$ & $<0.01$ & - & 0.91 & - \\
\hline
\end{tabular}


Conclusions These results confirm previous findings that NER1006 is a more effective bowel preparation than $\mathrm{NaP}+\mathrm{MgCit}$ and 2L PEG + Asc. If used routinely in large populations, the higher level of cleansing obtained with NER1006 will likely contribute towards increased lesion detection rates.

\section{REFERENCES}

1. Gastroenterology 2016;150(2):396-405.

2. Clin Gastroenterol Hepatol 2016;14(8):1155-62.

3. Gastroenterology 2016;150(4):S1269-70. Abstract Tu2084.

4. UEG Journal 2016;4(5):A1-156. OP375.

5. UEG Journal 2016;4(5):A157-A720. P1266

\section{PTH-068 ENDOSCOPIC CLOSURE OF ANTERIOR RESECTION ANASTOMOTIC DEHISCENCE USING PADLOCK CLIPS}

Javed Iqbal* ${ }^{*}$ Aleena Tariq, Sudeep Tanwar. Whipps Cross University Hospital, Barts Health NHS Trust, London, UK

\subsection{6/gutjnl-2018-BSGAbstracts.89}

Introduction Anastomotic leakage is one of the serious complications after anterior resection. The risk of colonic anastomotic leak after anterior resection has been reported to be as high as $23 \%$ in some published series. Herein, we present a case of successful endoscopic closure of anastomotic dehiscence using Padlock clipping system, which was used alongside the drainage of pelvic collection.

Method 43 days after developing anastomotic dehiscence post anterior resection, endoscopic Padlock clips were used to successfully close a $2 \mathrm{~cm}$ full thickness defect.

Results 73 years old male patient underwent laparoscopic anterior resection operation for moderately differentiated recto-sigmoid adenocarcinoma. He developed colonic anastomosis dehiscence on day 5 post-operatively. Emergency laparotomy was performed where drains were inserted with ileostomy formation while through-the-scope endoscopic clips deployed intraoperatively by the surgical team, were unable to successfully close the anastomotic defect. Patient was given parenteral nutrition post-procedure.

Two weeks later, faeculent fluid was noted in one of the drains, which suggested on-going leak. Repeat scan showed persistent extra-luminal leakage of the contrast with collection.

A flexible sigmoidoscopy was performed which identified a large $2 \mathrm{~cm}$ anastomotic site defect. This was then closed by the deployment of three Padlock clips along with three through-the-scope Resolution clips.

Conclusion Traditionally, surgical 'divert and drain' technique has been the mainstay of treatment for anastomotic leakage. Whereas endoscopic clips have been used successfully for the closure of anastomotic leaks, this case highlights that the Padlock system can be used successfully to close a chronic full thickness defect.

\section{PTH-069 VALIDATING A CLASSIFICATION SYSTEM USING ISCAN OPTICAL ENHANCEMENT FOR DETECTION OF EARLY BARRETT'S OESOPHAGUS NEOPLASIA}

\footnotetext{
${ }^{1}$ Martin Everson*, ${ }^{2}$ Raf Bisschops, ${ }^{3}$ Sachin Wani, Jacobo Ortiz Fernandez Sordo ${ }^{4}{ }^{5}$ Jose Miguel Esteban, ${ }^{1}$ Rami Sweis, ${ }^{1}$ Matt Banks, ${ }^{1}$ David Graham, 'Laurence Lovat, ${ }^{4}$ Krish Ragunath, ${ }^{1}$ Rehan Haidry. 'University College London Hospital, London, UK; ${ }^{2}$ University Hospitals Leuven, Leuven, Belgium; ${ }^{3}$ University of Colorado Anschutz Medical Center, Aurora, USA; ${ }^{4}$ NIHR Nottingham Digestive Diseases Biomedical Research Unit, Nottingham University Hospital, Nottingham, UK; ${ }^{5}$ Hospital Clínico San Carlos, Madrid, Spain
}

10.1136/gutjnl-2018-BSGAbstracts.90
Introduction Magnification endoscopy (ME) offers an additional tool for endoscopists undertaking BE surveillance to guide biopsies or direct endoscopic eradication therapy (EET) for early neoplasia. Mucosal patterns and oesophageal mucosal microvasculature can be interrogated. We propose a simple classification based on mucosal (M) and vascular (V) patterns, to differentiate dysplastic BE (DBE) mucosa from non-dysplastic (NDBE) mucosa. We assessed if magnification endoscopy paired with a new imaging platform, iScan OE (Pentax, Hoya, Japan) improves detection of early $\mathrm{BE}$ associated neoplasia. We validate our MV classification with this new platform.

Methods Patients undergoing BE surveillance from Jan 2016Nov 2017, were recruited from 3 international centres. ME was used to image the mucosa at up to $136 \mathrm{x}$ magnification. Endoscopists targeted normal and abnormal areas suspicious for neoplasia. Where possible, each area was imaged in HD-WLE and $\mathrm{OE}$ and matched histology was acquired from recorded areas. 7 experts blinded to histology classified HD images using our MV classification as NDBE or DBE. Mucosal patterns were graded as M1 (regular/gyric pits); M2 (irregular/featureless pits). Vascular patterns were graded as V1 (regular, normal calibre); V2 (dilated, irregular). M1V1 was considered NDBE; M2V1, M1V2 or M2V2 as DBE. Classifications were compared with histology. Accuracy, sensitivity, specificity were calculated. Krippendorf's alpha was used to assess interobserver agreement (IA). Results 54 patients were recruited: $63 \mathrm{HD}-\mathrm{WLE}$ and $90 \mathrm{OE}$ magnified images were obtained. The proportion of dysplastic areas imaged between WLE and OE was similar (29/63 vs 49/ $90 \mathrm{p}=\mathrm{NS})$. Using the MV classification, experts classified tissue as DBE or NDBE with $66.3 \%( \pm 0.05)$ accuracy using HDWLE vs $80.1 \%( \pm 0.02)$ with OE $[65-76 \%$ p $<0.001]$. Sensitivity improved from $81.2 \%( \pm 13)$ with $\mathrm{HD}-\mathrm{WLE}$ to $87.4 \%$ $( \pm 5.6)$ with OE [79-92\%] Specificity improved with OE; from $54.3 \%( \pm 17.6)$ in HD-WLE to $70 \%( \pm 4.4)$ in OE [62.76]. Both modalities showed fair IA between experts $(0.28$ in HD-WLE and 0.25 in OE).

Conclusion $\mathrm{ME}$ allows interrogation of the oesophageal mucosa and microvessels. We show that using iScan OE with $\mathrm{ME}$ in combination with the MV classification, confers a significant improvement in the accuracy, sensitivity and specificity of endoscopists in identifying early neoplasia, compared to HD-WLE alone.

\section{PTH-070 CORRELATION OF THE RECORDED IMAGE QUALITY INDEX (RIQI) SCORE WITH EXISTING COLONOSCOPY KEY PERFORMANCE INDICATORS}

Kate Edwards*, James Berrill, Joanna Hurley, Bee Lee, Neil Hawkes. Cwm Taf Uhb, Merthyr Tydfil, UK

\subsection{6/gutjnl-2018-BSGAbstracts.91}

Introduction The RIQI score (scale 0-10) has been validated as a measure of the quality of image recording during endoscopic examination. A high RIQI score (9-10) provides good clinical utility of the images to support clinical decision-making e.g. in MDTs. Taking high quality images requires good tip control to hold the correct focal length and time spent cleaning the mucosa. We aimed to assess the correlation between median RIQI scores and existing colonoscopy key performance indicators (KPIs) including the Caecal Intubation Rate (CIR), Polyp Detection Rate (PDR) and median colonoscopic withdrawal time (WDT). 